\title{
Changes of proteome components of Helicobacter pylori biofilms induced by serum starvation
}

\author{
CHUNHONG SHAO ${ }^{1,2^{*}}, \mathrm{YUNDONG} \mathrm{SUN}^{2 *}, \mathrm{NAWANG}^{2}, \mathrm{HAN}^{2} \mathrm{YU}^{2}$, \\ YABIN ZHOU ${ }^{2}$, CHUNYAN CHEN $^{3}$ and JIHUI JIA ${ }^{2}$ \\ ${ }^{1}$ Clinical Laboratory, Shandong Provincial Hospital Affiliated to Shandong University; \\ ${ }^{2}$ Department of Microbiology/Key Laboratory for Experimental Teratology of Chinese Ministry of Education, \\ School of Medicine, Shandong University; ${ }^{3}$ Department of Hematology, Qilu Hospital of \\ Shandong University, Jinan, Shandong 250012, P.R. China
}

Received May 27, 2013; Accepted September 26, 2013

DOI: $10.3892 / \mathrm{mmr} .2013 .1712$

\begin{abstract}
Biofilm is the adaptive living mechanism of Helicobacter pylori (H. pylori) during survival and propagation. Nutrient starvation is an environmental pressure for $H$. pylori in vivo and in vitro. Serum starvation effectively mimics the microenvironment in which $H$. pylori colonizes healthy humans who carry $H$.pylori and patients with chronic atrophic gastritis. In addition, it also mimics the in vitro environmental pressures of $H$. pylori. An H. pylori biofilm was successfully induced with serum starvation. To identify novel proteins associated with biofilm formation at the early stage in H. pylori, high-resolution 2-dementional gel electrophoresis was performed to obtain the proteome profiles of spiral $H$. pylori and early biofilm. Differential protein spots were identified using tandem matrix assisted laser desorption ionzation time of flight mass spectrometry, which revealed 35 proteins. These proteins are associated with various biological functions, including flagellar movement, bacterial virulence, signal transduction and regulation. To verify the results, the expression of cagA at the mRNA and protein levels was examined by fluorescence quantitative PCR and western blot analysis, respectively. This study indicates that $H$. pylori form biofilms by initiating multiple mechanisms involving a number of signaling pathways.
\end{abstract}

Correspondence to: Professor Jihui Jia, Department of Microbiology/Key Laboratory for Experimental Teratology of Chinese Ministry of Education, School of Medicine, Shandong University, 44 Wenhua Xi Road, Jinan, Shandong 250012, P.R. China E-mail: jiajihui@sdu.edu.cn

*Contributed equally

Key words: biofilm, comparative proteomics, Helicobacter pylori, serum starvation

\section{Introduction}

Helicobacter pylori (H. pylori) infection is a predominant cause of gastristis and peptic ulcer disease, as well as an early risk factor for gastric cancer. It is a globally epidemic pathogenic bacteria and more than half of the world's population has been infected with this bacterium. $H$. pylori infection is common; however, its precise mode of transmission has not yet been identified. The main reason for this is that the etiology of $H$. pylori has not been solved effectively. Therefore restrictng the formulation of prevention measures against $H$. pylori.

A biofilm is a multicellular layer of bacteria, which attaches to surfaces, interfaces or other cells and is embedded within a matrix of extracellular polymeric substances under stress conditions (1). Growth in a biofilm provides a number of advantages for bacteria, including enhanced power of resistance to environmental stresses as well as to host defenses, which leads to serious clinical problems, particularly chronic and refractory infection. The biofilm of $H$. pylori has been observed in the water system, including drinking water, groundwater and sewage $(2,3)$ and also on the human gastric mucosal epithelium (4,5). A number of studies have been performed on $H$. pylori biofilms $(6,7)$. Thus, biofilms are hypothesized to be another adaptive survival mode of $H$. pylori. In conventional experiments, $H$. pylori is cultured in a rich medium containing fetal bovine serum (FBS). However, this may not effectively imitate the in vitro and in vivo survival environment of $H$. pylori. Nutrient starvation is a common environmental pressure that $H$. pylori is faced with. Serum starvation effectively mimics the microenvironment $H$. pylori within healthy human carriers of $H$. pylori and patients with chronic atrophic gastritis (8) and also the in vitro pressure environment pressure that $H$. pylori is subjected to. The current study identified that $H$. pylori adhered to each other and formed a population structure similar to that of a bacterial biofilm by removing serum from medium. Williams et al (9) observed that serum significantly affects the movement, morphology and adhesion of $H$. pylori and may result in agglutination in $1 \%$ serum concentration. Campylobacter jejuni also agglutinate in nutrient-limiting conditions. Reeser et al (10) observed that the autoagglutinated structure is a biofilm by analyzing its extracellular polymer matrix. 
The formation of a biofilm is a complex and dynamic process. Under adverse conditions, the bacteria induce a unique set of genes, adhere to each other and form structural groups or subgroups. During biofilm formation, each link may be regarded as a key point to treat infection and drug design target, particularly at the early stage. In the present study, proteomic technologies were used to analyze the proteomic expression profiles of $H$. pylori biofilms at an early stage, and aimed to reveal the proteins and signaling molecules involved in $H$. pylori biofilm formation and identify novel pathogenic factors and antibacterial targets.

\section{Materials and methods}

Bacterial strain and culture conditions. The H.pylori strain 26695 was provided by Dr Zhang Jianzhong from the Chinese Disease Control and Prevention Center. It was cultured in Brucella broth with $10 \%$ FBS with 120 rpm agitation under microaerobic conditions $\left(5 \% \mathrm{O}_{2}, 10 \% \mathrm{CO}_{2}, 85 \% \mathrm{~N}_{2}\right.$, v/v) at $37^{\circ} \mathrm{C}$. The same quantity of exponentially growing $H$. pylori was resuspended in Brucella broth with and without $10 \%$ FBS. Bacterial morphological changes were then observed using Gram staining at intervals of $2 \mathrm{~h}$ until the autoagglutinated structure formed. The initial time of biofilm formation was recorded. Subsequently, the planktonic $H$. pylori and the biofilm were fixed with $2.5 \%$ glutaraldehyde solution for $>2$ h. The critical-point dried sample was observed with a Hitachi S-520 scanning electron microscope (Hitachi Limited Company, Tokyo, Japan).

2-dimentional gel electrophoresis (2-DE) and image analysis. The planktonic $H$. pylori and its early biofilm cultured with and without $10 \%$ FBS for $4 \mathrm{~h}$ were harvested by centrifugation at $5000 \mathrm{x} \mathrm{g}$ for $10 \mathrm{~min}$ at $4^{\circ} \mathrm{C}$ and washed three times with ice-cold PBS (pH 7.2). The pellets were solubilized in lysis buffer containing $8 \mathrm{M}$ urea, $2 \mathrm{M}$ thiourea, 4\% CHAPS, $1 \%$ DTT, $1 \%$ pharmalyte (pH range, $3-10$ ), $1 \%$ protease inhibitor and 1\% nuclease mix (Amersham Pharmacia Biotech, Piscatawway, NJ, USA). Following sonication, the solution was centrifugated at $20,000 \mathrm{x}$ g for $60 \mathrm{~min}$ at $4^{\circ} \mathrm{C}$ to remove cell debris. Protein concentrations were determined using the Bradford method and proteins were stored at $-80^{\circ} \mathrm{C}$. The $2-\mathrm{DE}$ analysis was performed as described previously (11). The SDS gels were silver-stained and the protein patterns were scanned using an ImageScannerII (Amersham Pharmacia Biotech). The gels were analyzed by ImageMaster 2D Elite 5.0 (Amersham Pharmacia Biotech) to determine differentially expressed protein spots whose vol\% ratio increased $>2$-fold $(\mathrm{P}<0.05)$.

In-gel digestion and matrix assisted laser desorption ionzation time of flight mass spectrometry (MALDI-TOF-TOF $M S)$. Differentially expressed protein spots were excised, tryptic digested and identified with a 4700 MALDI-TOF-TOF proteomic system (Applied Biosystems) as described previously (11). The MS and MS/MS spectra were analyzed with a 50 ppm mass tolerance by GPS Explorer V.2.0.1 and Mascot V1.9 based on the NCBI and SWISSPROT databases.

Quantitative PCR . Total RNA was isolated from three independent cultures of planktonic $H$. pylori and biofilm at early
Table I. Primers used in the study

\begin{tabular}{lc}
\hline Primers & Sequence $\left(5^{\prime}-3^{\prime}\right)$ \\
\hline cagA Forward & GCTTACCGCCTGAAGCTAGG \\
cagA Reverse & CCTTTCTCACCACCTGCTATG \\
16S rRNA Forward & GCTCTTTACGCCCAGTGATTC \\
16S rRNA Reverse & GCGTGGAGGATGAAGGTTTT \\
\hline
\end{tabular}

stages using TRIzol reagent (Invitrogen Life Technologies, Carlsbad, CA, USA) according to the manufacturer's instructions. The quantity was measured by absorbance at $260 \mathrm{~nm}$. Subsequently, $4 \mu \mathrm{g}$ total template RNA was reverse transcribed into cDNA using M-MLV reverse transcriptase and random hexamer primer (MBI). The primers for PCR are listed in Table I. The 16S rRNA gene served as the endogenous control. PCR was performed as described previously (12). The operating conditions were as follows: One cycle at $50^{\circ} \mathrm{C}$ for $2 \mathrm{~min}$, one cycle at $95^{\circ} \mathrm{C}$ for $10 \mathrm{~min}, 40$ cycles at $95^{\circ} \mathrm{C}$ for $15 \mathrm{sec}$ and $60^{\circ} \mathrm{C}$ for $1 \mathrm{~min}$. Gene expression was quantified by the comparative threshold cycle $(\mathrm{Ct})$ method and normalized to the quantity of $16 \mathrm{~S}$ rRNA cDNA in each sample. The relative quantity of the target was calculated by the $\Delta \Delta \mathrm{CT}$-method.

Western blot analysis. Protein samples were separated by sodium dodecyl sulfate (SDS)-polyacrylamide gel electrophoresis (8\% SDS-acrylamide gels) and transferred to a nitrocellulose membrane. The membranes were incubated in blocking buffer [TBS containing $0.1 \%$ Tween-20 (TBST) and $5 \%$ non-fat powdered milk] for $1.5 \mathrm{~h}$ and immunoblotted for $1.5 \mathrm{~h}$ with antibodies against $\beta$-actin or C-CagA (Santa Cruz Biotechnology, Inc. Santa Cruz, CA, USA). Membranes were washed three times with the TBST solution and incubated with the corresponding antibodies conjugated with horseradish peroxidase for $50 \mathrm{~min}$. The washed membranes were developed by the Chemilucent ECL Detection system (Millipore, Billerica, MA, USA).

\section{Results and Discussion}

In recent years, increasing evidence has indicated that bacterial biofilms are important in infectious diseases. Biofilms effectively protect bacteria from external environmental damage and are an effective strategy to adapt to environmental pressures in vivo and in vitro. As one of the predominant pathogens affecting humans, $H$. pylori was subjected to various types of adverse conditions, including nutrient starvation. To produce the survival environment $H$. pylori generated when under starvation stress, exponentially growing $H$. pylori 26695 (Fig. 1A) was inoculated into medium without FBS. Following $4 \mathrm{~h}$, the bacteria began to adhere to each other and formed a subgroup structure (Fig. 1B). Typical community structures were observed following $12 \mathrm{~h}$ starvation and a number of $H$. pylori changed to a dormant coccoid form (Fig. 1C). Thus, a biofilm of H. pylori was successfully induced with serum starvation.

To identify novel proteins associated with $H$. pylori biofilm formation, high-resolution 2-DE was performed to obtain the 

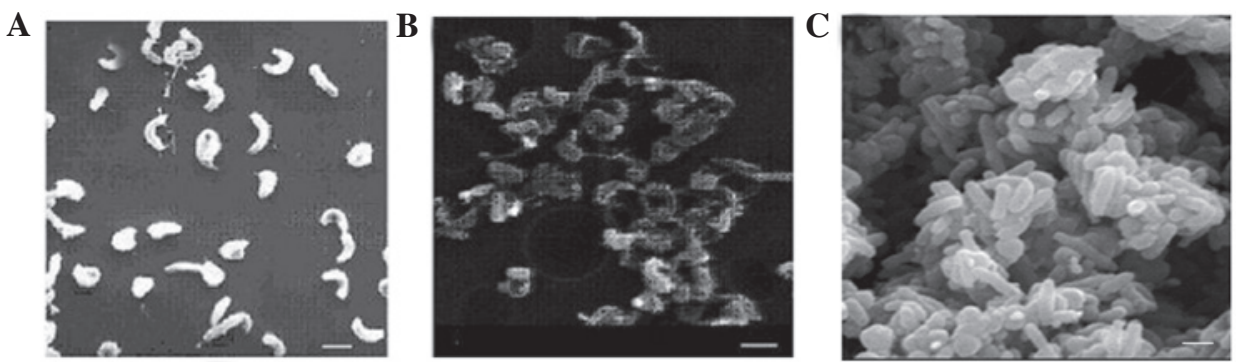

Figure 1. Scanning electron microscopy of floating unicellular $H$. pylori and its biofilm. (A) Floating unicellular $H$. pylori cultured with $10 \%$ FBS; (B) $H$. pylori biofilm cultured without FBS for $4 \mathrm{~h}$ and (C) H.pylori biofilm cultured without FBS for $12 \mathrm{~h}$. Scale bar, $1 \mu \mathrm{m}$. H. pylori, Helicobacter pylori; FBS, fetal bovine serum.

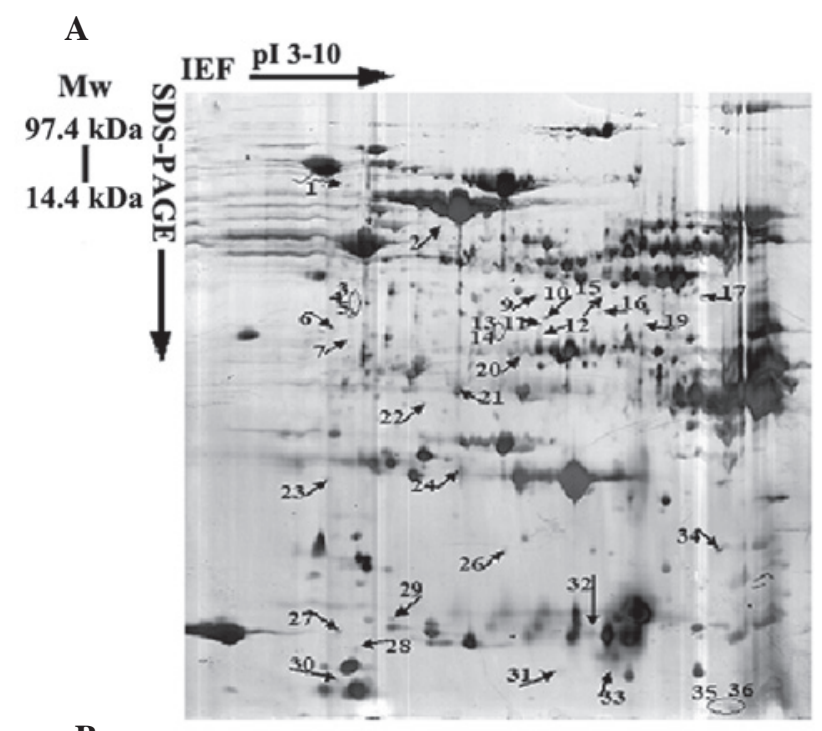

B

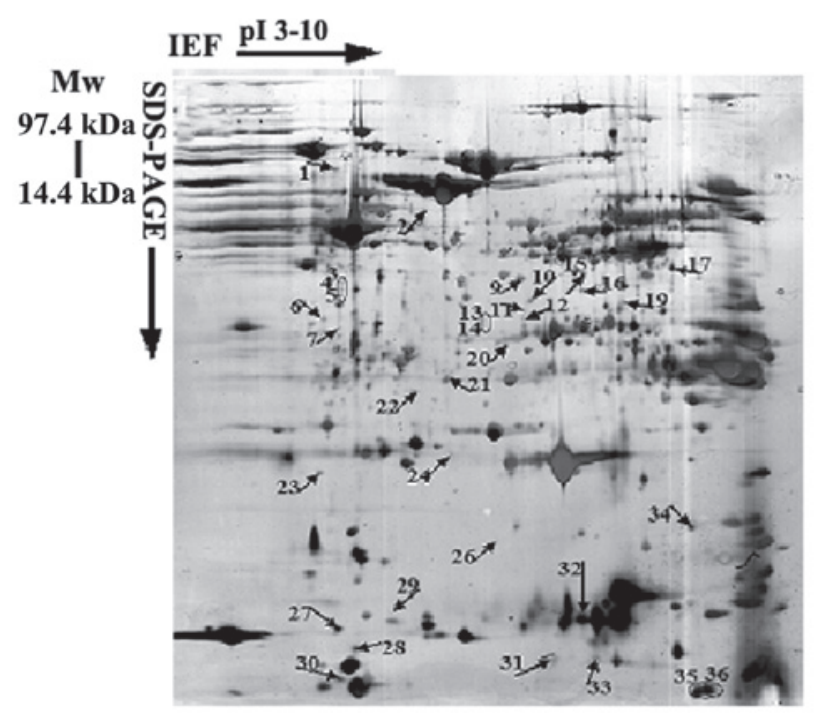

Figure 2. 2-DE profiles of whole cell proteins from (A) floating unicellular $H$. pylori and (B) its early biofilm following serum starvation for 4 h. H. pylori, Helicobacter pylori.

proteome profiles of spiral $H$. pylori and its early biofilm, which was induced by $4 \mathrm{~h}$ of starvation (Fig. 2). 2-DE analysis was repeated in triplicate using independently grown cultures. The comparison of the protein patterns of these two forms of $H$. pylori revealed that 36 spots exhibited different levels of expression ( $>2$-fold, $\mathrm{P}<0.05$ ). These spots were subjected to

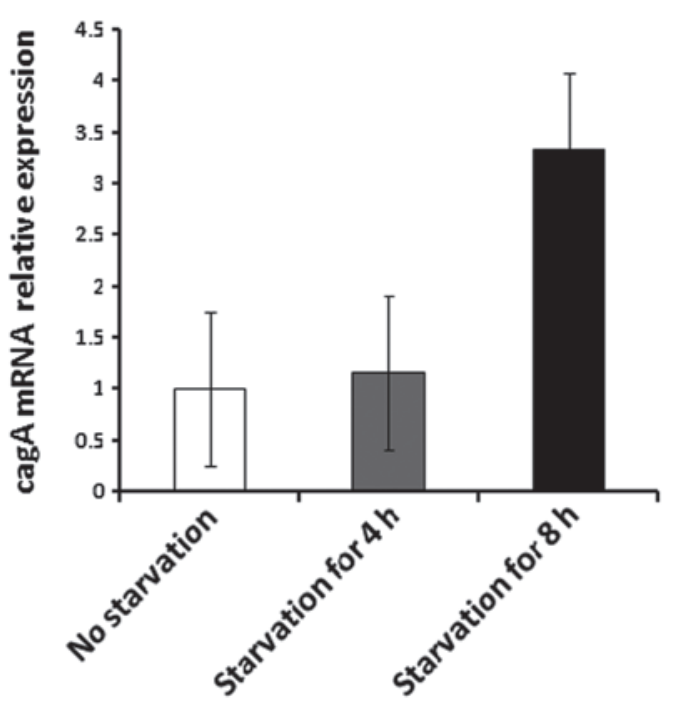

Figure 3. Expression of cagA in $H$. pylori cultured with $10 \%$ FBS and without FBS for $4 \mathrm{~h}$ and $8 \mathrm{~h}$ observed through quantitative PCR. H. pylori, Helicobacter pylori; FBS, fetal bovine serum.

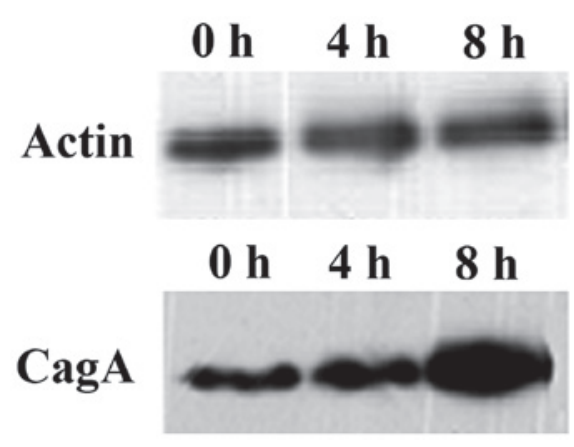

Figure 4. CagA protein expression in $H$. pylori cultured with $10 \%$ FBS and without FBS for $4 \mathrm{~h}$ and $8 \mathrm{~h}$ detected by western blot analysis. H. pylori, Helicobacter pylori; FBS, fetal bovine serum.

MALDI-TOF-TOF MS analysis to determine their putative genes and infer their functions. A total of 35 proteins with different functions were identified (Table II); of which 29 were upregulated and six were downregulated in the early biofilm of H. pylori. These proteins belong to diverse functional classes, including movement, virulence, energy metabolism, regulator and chaperone proteins. This suggests that $H$. pylori invokes a multi-mechanism response to adapt to morphological changes under starvation stress. 
Table II. Summary of protein spots showing altered expression between planktonic H. pylori and its early biofilm.

\begin{tabular}{|c|c|c|c|c|c|}
\hline $\begin{array}{l}\text { Spot } \\
\text { number }\end{array}$ & Protein (gene) & $\begin{array}{l}\text { TIGR ORF } \\
\text { number }^{b}\end{array}$ & $\mathrm{Gi}$ & Top score & $\%$ vol ratio ${ }^{c}$ \\
\hline 1 & Cag pathogenicity island protein (cag26) & Hp0547 & 2313664 & 139 & 2.89 \\
\hline 2 & Flagellar capping protein(fliD) & Hp0752 & 2313869 & 85 & 2.21 \\
\hline 3 & Aconitase B (acnB) & Hр0779 & 2313908 & 105 & 2.36 \\
\hline 4 & Aconitase B (acnB) & Hр0779 & 2313908 & 133 & 2.13 \\
\hline 6 & Type I restriction enzyme R protein (hsdR) & Hp0846 & 2313977 & 46 & 2.05 \\
\hline 6 & Urease $\beta$-subunit (urea amidohydrolase) (ureB) & Hр0072 & 2313153 & 45 & 2.05 \\
\hline 6 & Arginase (rocF) & Hp1399 & 2314565 & 50 & 2.05 \\
\hline 7 & H. pylori predicted coding region HP0013 & Hр0013 & 2313087 & 64 & 2.02 \\
\hline 9 & Transaldolase (tal) & Hp1495 & 2314674 & 175 & 2.04 \\
\hline 10 & Holliday junction DNA helicase (ruvB) & Hp1059 & 2314203 & 161 & 2.04 \\
\hline 10 & GTP-binding protein, fusA-homolog (yihK) & Hр0480 & 2313589 & 75 & 2.04 \\
\hline 11 & UDP-N-acetylenolpyruvoylglucosamine reductase (murB) & Hp1418 & 2314592 & 69 & 2.15 \\
\hline 12 & Chaperone and heat shock protein (groEL) & Hр0010 & 2313084 & 106 & 7.45 \\
\hline 13 & Poly E-rich protein & Hp0322 & 2313421 & 64 & 2.14 \\
\hline 14 & DNA-directed RNA polymerase, $\beta$-subunit (rpoB) & Hp1198 & 2314357 & 64 & 2.26 \\
\hline 15 & GTP-binding protein (obg) & Hp0303 & 2313401 & 128 & 3.24 \\
\hline 15 & UDP-N-acetylenolpyruvoylglucosamine reductase (murB) & Hp1418 & 2314592 & 61 & 3.24 \\
\hline 16 & Delta-aminolevulinic acid dehydratase (hemB) & Hp0163 & 2313250 & 111 & 2.13 \\
\hline 17 & Aspartate-semialdehyde dehydrogenase (asd) & Hp1189 & 2314350 & 257 & 2.31 \\
\hline 19 & Aspartyl-tRNA synthetase (aspS) & Нp0617 & 2313739 & 65 & 3.16 \\
\hline 20 & H. pylori predicted coding region HP0958 & Hp0958 & 2314102 & 90 & 4.76 \\
\hline 21 & Conserved hypothetical protein & Hp1588 & 2314773 & 73 & 0.32 \\
\hline 21 & 3-oxoadipate coA-transferase subunit A (yxjD) & Нp0691 & 2313815 & 68 & 0.32 \\
\hline 22 & Response regulator (ompR) & Hp0166 & 2313252 & 105 & 2.07 \\
\hline 23 & H. pylori predicted coding region HP0406 & Hр0406 & 2313513 & 131 & 2.01 \\
\hline 24 & Alkyl hydroperoxide reductase (AhpC) & Hp1563 & 2314747 & 146 & 0.31 \\
\hline 26 & Cag pathogenicity island protein (cag24) & Hр0504 & 2313660 & 77 & 0.44 \\
\hline 26 & Translation elongation factor EF-Tu (tufB) & Hp1205 & 2314366 & 45 & 0.44 \\
\hline 27 & Conserved hypothetical protein & Hp1046 & 2314193 & 76 & 2.57 \\
\hline 27 & Hemolysin secretion protein precursor (hylB) & Hр0599 & 2313716 & 49 & 2.57 \\
\hline 28 & Riboflavin synthase beta chain (ribE) & Hp0002 & 2313079 & 121 & 2.66 \\
\hline 29 & Nonheme iron-containing ferritin (pfr) & Hр0653 & 2313771 & 82 & 0.03 \\
\hline 30 & Hydrogenase expression/formation protein (hypA) & Нp0869 & 2313996 & 104 & 4.37 \\
\hline 31 & H. pylori predicted coding region HP1377 & Hp1377 & 2314555 & 111 & 2.55 \\
\hline 32 & Co-chaperone (groES) & Hp0011 & 2313085 & 82 & 5.08 \\
\hline 34 & H. pylori predicted coding region HP1029 & Hp1029 & 2314185 & 142 & 2.41 \\
\hline 35 & Thioredoxin & Hp1458 & 2314636 & 97 & 81.74 \\
\hline 36 & Thioredoxin & Hp1458 & 2314636 & 126 & 2.07 \\
\hline
\end{tabular}

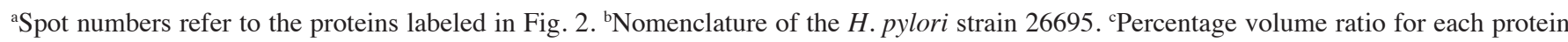
derived from floating unicellular H. pylori with respect to the protein derived from the early biofilm. H. pylori, Helicobacter pylori.

The adhesion of bacteria is the early stages of biofilm formation and the flagellar movement are important in this process. The present study identified that the flagellar filament capping protein (HAP2) was increased in the early biofilm of H.pylori. This protein is encoded by the fliD gene, constitutes the flagellar hook together with HAP1 and HAP3, and is involved in the flagellar assembly of $H$. pylori. Martin et al observed that proteins associated with flagella exhibited higher expres- sion in the biofilm of Campylobacter and that the deletion of flagellar genes may seriously affect the biofilm formation of bacteria $(13,10)$. In Escherichia coli (E. coli), flagellar motility may significantly affect the three-dimensional structure of the biofilm (14). In addition, aconitase was observed to be upregulated in H. pylori early biofilms. The structural analysis identified that this protein contained a domain similar to the HEAT domain, which mediates the interaction between 
proteins. In E. coli and Bacillus subtilis, the HEAT protein has been confirmed to have a dual function and regulate flagellar movement as a post-transcriptional regulator under iron and oxygen conditions $(15,16)$. Under serum starvation conditions, the overexpression of aconitase in $H$. pylori is hypothesized to be involved in biofilm formation by the promotion of flagellar movement. However, this requires further study to be confirmed.

In present study, the cagA protein encoded by cag pathogenicity islands was identified to be induced in $H$. pylori biofilms. As an important virulence factor of $H$. pylori, this protein is injected into gastric mucosa epithelial cells through the type IV secretion system when the cagA positive $H$. pylori infect the individual. CagA is then localized in the inner surface of the plasma membrane and results in a series of changes, including excessive proliferation and phenotypic change, such as extended 'hummingbird' morphology (17). To confirm the results, the expression of cagA was examined at the mRNA and protein levels by quantitative PCR and western blot analysis, respectively. The results showed that the expression of cagA was not significantly changed following $4 \mathrm{~h}$ of starvation. This may be due to higher resolution of the 2-DE compared with 1-D SDS. However, after $8 \mathrm{~h}$ cagA expression was upregulated significantly (Figs. 3 and 4). Thus the upregulation of this protein was hypothesized to aid in the improvement of the viability of $H$. pylori under adverse environments.

UspA is a conservative and integral promoter of a number of stress-associated proteins in a variety of bacteria. The $\beta$-subunit of RNA polymerase positively regulates the expression of UspA under a number of environmental pressures, including starvation. Thus, a variety of stress-related proteins show higher expression levels and bacterial biofilms have strong resistance to the outside pressure (18). In the current study, the $\beta$-subunit of RNA polymerase and two chaperone protein GroES and GroEL of $H$. pylori were observed to be overexpressed under serum starvation. In addition, two oxidative stress-related proteins, alkyl hydroperoxide reductase and thioredoxin, were identified to have a high level of expression in the biofilm of $H$. pylori. This is consistent with the biofilms of E. coli and Campylobacter $(13,19)$.

The two-component system is a common signal transduction mechanism in bacterial responses to various stresses. This system is composed of two types of proteins, a histidine kinase and a response regulator. These two components exchange signals by phosphate transfer. Two-component systems are involved in the biofilm formation of $E$. coli, Pseudomonas aeruginosa and other bacteria (20,21). For example, the EnvZ/OmpR two-component system in E. coli promotes biofilm formation by regulating the expression of proteins associated with bacterial fimbriae to enhance its adhesion (22). In the present study, the response regulator Hp0166 was observed to be induced in $H$. pylori biofilms. A previous study also demonstrated that the Ars two-component system is involved in $H$. pylori responses to acid stress by regulating the expression of a variety of genes (23).

In addition, the current study also identified that the expression of other proteins changed in H. pylori biofilms. The expression of urease and arginase was upregulated. These two proteins generate ammonia in the process of amino acid metabolism, which is essential to biofilm formation to main- tain the $\mathrm{pH}$ balance. The accessory protein of urease, ureE, is highly expressed in Staphylococcus aureus biofilms (24). Peptidoglycan is a predominant component of the cell walls of bacteria, UDP-N acetylene alcohol acid glucosamine reductase is an enzyme that is required in peptidoglycan biosynthesis. It catalyzes the reduction of UDPN GlcNAc to UDPN acetyl muramic acid (25). This protein was overexpressed in $H$. pylori biofilms and it is consistent with a previous study of Staphylococcus aureus (14). The function of peptidoglycan remains unclear, but it was observed that fracture of the peptidoglycan may promote the formation of bacterial biofilms (26). In addition, this study also showed that the expression of a number of other unknown proteins, including Hp1377 and Hp1029, changed in H. pylori biofilm formation. However, this also requires further study.

In the present study, H. pylori biofilm formation was induced successfully by serum starvation and its proteome was analyzed using proteomic methods. This study showed that $\geq 35$ proteins are involved in biofilm formation. These proteins are associated with a number of types of biological functions, including flagellar movement, bacterial virulence, signal transduction and regulation. Such results showed that $H$. pylori biofilms are formed through multiple mechanisms involving numerous signal pathways. These findings may provide valuable information in understanding the survival mechanism of this bacterium in animals and humans.

\section{Acknowledgements}

This study was supported by grants from the National Natural Science Foundation of China (grant nos. 81171536 and 30800037) and the Science Foundation of Shandong Province, PR China (grant nos. ZR2009CZ001 and ZR2009CM002).

\section{References}

1. Donlan RM and Costerton JW: Biofilms: survival mechanisms of clinically relevant microorganisms. Clin Microbiol Rev 15: 167-193, 2002.

2. Moreno Y and Ferrús MA: Specific detection of cultivable Helicobacter pylori cells from wastewater treatment plants. Helicobacter 17: 327-332, 2012.

3. Braganra SM, Azevedo NF, Simões LC, Keevil CW and Vieira MJ: Use of fluorescent in situ hybridisation for the visualisation of Helicobacter pylori in real drinking water biofilms. Water Sci Technol 55: 387-393, 2007.

4. Cammarota G, Sanguinetti M, Gallo A and Posteraro B: Review article: biofilm formation by Helicobacter pylori as a target for eradication of resistant infection. Aliment Pharmacol Ther 36: 222-230, 2012

5. Carron MA, Tran VR, Sugawa C and Coticchia JM: Identification of Helicobacter pylori biofilms in human gastric mucosa. J Gastrointest Surg 10: 712-717, 2006.

6. Yang FL, Hassanbhai AM, Chen HY, Huang ZY, Lin TL, Wu SH and Ho B: Proteomannans in biofilm of Helicobacter pylori ATCC 43504. Helicobacter 16: 89-98, 2011.

7. GrandeR,DiGiulio M,BessaLJ,DiCampliE,BaffoniM,Guarnieri S and Cellini L: Extracellular DNA in Helicobacter pylori biofilm: a backstairs rumour. J Appl Microbiol 110: 490-498, 2011.

8. Testerman, TL, Conn PB, Mobley HL and McGee DJ: Nutritional requirements and antibiotic resistance patterns of Helicobacter species in chemically defined media. J Clin Microbiol 44: 1650-1658, 2006.

9. Williams JC, McInnis KA and Testerman TL: Adherence of Helicobacter pylori to abiotic surfaces is influenced by serum. Appl Environ Microbiol 74: 1255-1258, 2008. 
10. Reeser RJ, Medler RT, Billington SJ, Jost BH and Joens LA Characterization of Campylobacter jejuni biofilms under defined growth conditions. Appl Environ Microbiol 73: 1908-1913, 2007.

11. Shao C, Zhang Q, Sun Y, et al: Helicobacter pylori protein response to human bile stress. J Med Microbiol. 57: 151-158, 2008.

12. Shao C, Zhou Y, Sun Y, et al: Analysis of aztreonam-inducing proteome changes in nondividing filamentous Helicobacter pylori. Curr Microbiol 2012 65: 108-115, 2012.

13. Kalmokoff M, Lanthier P, Tremblay TL, et al: Proteomic analysis of Campylobacter jejuni 11168 biofilms reveals a role for the motility complex in biofilm formation. J Bacteriol 188 : 4312-4320, 2006.

14. Wood TK, González Barrios AF, Herzberg M and Lee J: Motility influences biofilm architecture in Escherichia coli. Appl Microbiol Biotechnol 72: 361-367, 2006.

15. Tang Y, Guest JR, Artymiuk PJ and Green J: Switching aconitase $\mathrm{B}$ between catalytic and regulatory modes involves iron-dependent dimer formation. Mol Microbiol 56: 1149-1158, 2005.

16. Tang Y, Guest JR, Artymiuk PJ, Read RC and Green J: Post-transcriptional regulation of bacterial motility by aconitase proteins. Mol Microbiol 51: 1817-1826, 2004.

17. Backert S, Moese S, Selbach M, Brinkmann V and Meyer TF Phosphorylation of tyrosine 972 of the Helicobacter pylori CagA protein is essential for induction of a scattering phenotype in gastric epithelial cells. Mol Microbiol 42: 631-644, 2001

18. Kvint K, Hosbond C, Farewell A, Nybroe O and Nyström T: Emergency derepression: stringency allows RNA polymerase to override negative control by an active repressor. Mol Microbiol 35: 435-443, 2000
19. Schembri MA, Kjaergaard K and Klemm P: Global gene expression in Escherichia coli biofilms. Mol Microbiol 48: 253-267, 2003.

20. Li YH, Lau PC, Tang N, Svensäter G, Ellen RP and Cvitkovitch DG: Novel two-component regulatory system involved in biofilm formation and acid resistance in Streptococcus mutans. J Bacteriol 184: 6333-6342, 2002.

21. Otto K and Silhavy TJ: Surface sensing and adhesion of Escherichia coli controlled by the Cpx-signaling pathway. Proc Natl Acad Sci USA 99: 2287-2292, 2002.

22. Prigent-Combaret C, Brombacher E, Vidal O, Ambert A, Lejeune P, Landini P and Dorel C: Complex regulatory network controls initial adhesion and biofilm formation in Escherichia coli via regulation of the csgD gene. J Bacteriol 183: 7213-7223, 2001.

23. Wen Y, Feng J, Scott DR, Marcus EA and Sachs G: Involvement of the HP0165-HP0166 two-component system in expression of some acidic-pH-upregulated genes of Helicobacter pylori. J Bacteriol 188: 1750-1761, 2006

24. Resch A, Leicht S, Saric M, Pásztor L, Jakob A, Götz F and Nordheim A: Comparative proteome analysis of Staphylococcus aureus biofilm and planktonic cells and correlation with transcriptome profiling. Proteomics 6: 1867-1877, 2006.

25. Andres CJ, Bronson JJ, D'Andrea SV, et al: 4-Thiazolidinones: novel inhibitors of the bacterial enzyme MurB. Bioorg Med Chem Lett 10: 715-717, 2000.

26. Mercier C, Durrieu C, Briandet R, Domakova E, Tremblay J, Buist $\mathrm{G}$ and Kulakauskas S: Positive role of peptidoglycan breaks in lactococcal biofilm formation. Mol Microbiol 46: 235-243, 2002 . 